CLINICAL STUDY

\title{
Elevated plasma levels of SPARC in patients with newly diagnosed type 2 diabetes mellitus
}

\author{
Dandong $\mathrm{Wu}^{*}$, Ling $\mathrm{Li}^{1, *}$, Mengliu Yang, Hua Liu ${ }^{2}$ and Gangyi Yang \\ Department of Endocrinology, The Second Affiliated Hospital, Chongqing Medical University, 76 Linjiang Road, Yuzhong District, 400010 Chongqing, \\ China, ${ }^{1}$ The Key Laboratory of Laboratory Medical Diagnostics in the Ministry of Education and Department of Clinical Biochemistry, Chongqing Medical \\ University, Chongqing, China and ${ }^{2}$ Department of Pediatrics, University of Mississippi Medical Center, Jackson, Mississippi, USA \\ (Correspondence should be addressed to G Yang; Email: gangyiyang@yahoo.com.cn) \\ *(L Li and D Wu contributed equally to this work)
}

\begin{abstract}
Objective: Secreted protein acidic and rich in cysteine (SPARC) also known as BM-40 which has been studied in various pathological conditions, has recently been suggested as a key player in the pathology of obesity and type 2 diabetes mellitus (T2DM). However, there are few studies on putative pathophysiologic roles of SPARC in glucose metabolism. The aim of this study was to determine whether plasma SPARC concentrations were altered in subjects with different glucose metabolic conditions and to investigate the affecting factors.

Design and methods: In this study, 54 newly diagnosed T2DM subjects, 53 subjects with impaired glucose regulation (IGR), and 53 normal subjects (body mass index (BMI): $24.98 \pm 3.75$ vs 24.70 \pm 2.78 and $24.53 \pm 3.66 \mathrm{~kg} / \mathrm{m}^{2}, P>0.05$ ) were enrolled. Plasma SPARC levels were measured with an ELISA under overnight fasting conditions. The relationships between plasma SPARC and several metabolic factors, such as BMI, blood lipids, blood glucose, plasma insulin levels, and other factors were also assessed.

Results: SPARC levels were higher in subjects with T2DM compared with IGR and control subjects $(16.74 \pm 6.99$ vs $14.04 \pm 8.03 \mu \mathrm{g} / \mathrm{l}, P<0.05$ and $16.74 \pm 6.99$ vs $11.72 \pm 4.47 \mu \mathrm{g} / \mathrm{l}, P<0.01)$. However, there was no difference in plasma SPARC levels between IGR subjects and the controls. Plasma SPARC levels correlated positively with BMI, the percentage of fat, triglyceride, fasting plasma insulin, $2 \mathrm{~h}$ plasma insulin after a glucose load, and the homeostasis model assessment of insulin resistance in simple regression analysis.
\end{abstract}

Conclusion: The present work indicates a potential link between SPARC and the pathogenesis of T2DM.

European Journal of Endocrinology 165 597-601

\section{Introduction}

Secreted protein acidic and rich in cysteine (SPARC), also known as BM-40, first identified in bone, is also known as osteonectin (1). It is expressed in most tissues but, in obesity, adipose tissue cells are the major source of circulating SPARC (2). As a multifunctional glycoprotein binding calcium, collagen and hydroxyapatite (3), SPARC is an extracellular regulatory macromolecule involved in osteogenesis, angiogenesis, wound healing, tumorigenesis, hepatic and renal fibrosis (4-6). Evidence suggests that a higher level of SPARC appears to inhibit the expansion of abdominal subcutaneous adipose tissue (7), probably by increasing ectopic lipid stores in other tissues such as liver or skeletal muscle resulting in predisposition to insulin resistance (IR). Expression of SPARC is markedly elevated in the adipose tissue in both obese (OB) mice and human individuals $(2,8)$, and is positively regulated by weight change, calorie intake, insulin, and leptin. There is an association between SPARC and IR in human individuals, independent of obesity (2). In addition, SPARC has been implicated in the pathogenesis of diabetes complications such as retinopathy and nephropathy (9-12). However, there is currently little information on the variation in circulating SPARC levels in subjects with different glucose metabolic conditions.

In this study, plasma levels of SPARC in normal, impaired glucose regulation (IGR) and type 2 diabetes mellitus (T2DM) subjects were evaluated to better appreciate its clinical importance. The relationships between circulating plasma SPARC levels and body mass index (BMI), blood lipids, blood glucose, plasma insulin, and homeostasis model assessment of IR $\left(\mathrm{HOMA}_{\mathrm{IR}}\right)$, as well as other factors, were also investigated. 


\section{Materials and methods}

\section{Subjects}

In this study, 160 Chinese volunteers were enrolled, including 54 subjects with T2DM (29 men and 25 women), 53 subjects with IGR ( 25 men and 28 women, including 21 subjects with impaired fasting glucose and 32 subjects with impaired glucose tolerance), and 53 control subjects with normal glucose tolerance ( 26 men and 27 women). All these subjects with T2DM and IGR were diagnosed according to the criteria of the American Diabetes Association (Diabetes Care 1997). Based on BMI, subjects were divided into two groups: a normal weight (NW; BMI $<25 \mathrm{~kg} / \mathrm{m}^{2}, 86$ subjects) and an overweight $(\mathrm{OW}) / \mathrm{OB}\left(\mathrm{BMI}>25 \mathrm{~kg} / \mathrm{m}^{2}, 74\right.$ subjects) groups. Subjects were excluded if they had T1DM, acute or chronic infectious disease, hypertension, heart failure, hepatic or renal disease, and long-term diabetic complications. Subjects were newly diagnosed with T2DM or IGR, and had not been treated with oral antihyperglycemic agents or insulin. None of the control subjects were taking any medication affecting blood glucose tolerance. This study was approved by the Human Research Ethics Committee of Chongqing Medical University. An informed consent was obtained from all participants in this study.

\section{Anthropometry and blood samples}

Anthropometric measurement was performed in the morning, before breakfast, with the subjects wearing light clothing, without shoes. Body weight and height were measured in all subjects using a scale and a wall mounted stadiometer to the nearest $0.5 \mathrm{~kg}$ and $0.5 \mathrm{~cm}$ respectively. Waist and hip circumferences were measured, and the waist-to-hip ratio (WHR) was calculated. A $75 \mathrm{~g}$ oral glucose tolerance test was conducted after an 8-12 h overnight fast. Venous blood samples were obtained at $0,30,60$, and $120 \mathrm{~min}$ after the glucose load. Plasma glucose and HbAlc were measured by the glucose oxidase method and isoelectric focusing respectively. Serum samples were frozen and stored at $-70{ }^{\circ} \mathrm{C}$ for the measurements of SPARC, insulin, free fatty acid (FFA), and blood fat levels.

\section{Analytical procedure}

SPARC was evaluated using a commercially available ELISA Kit (Adipobiotech, Beijing, China). The linear range of the assay was $3.2-2000 \mathrm{ng} / \mathrm{ml}$ for SPARC. The interand intra-assay coefficients of variation were 8 and $4 \%$ for SPARC respectively (all data and specifications of the SPARC ELISA were derived from the manufacturer). Plasma insulin was measured by RIA using human insulin as standard (Institute of Atomic Energy, China). FFAs were measured with a commercial assay kit (Randox Laboratories Ltd, Antrim, UK). triglyceride (TG), total cholesterol, high-density lipoprotein cholesterol, and low-density lipoprotein cholesterol concentrations were determined enzymatically. The HOMA $\mathrm{IR}_{\mathrm{IR}}$ and the HOMA of insulin secretion $\left(\mathrm{HOMA}_{\mathrm{IS}}\right)$ were calculated from fasting insulin and glucose levels with following equations: $\mathrm{HOMA}_{\mathrm{IR}}=$ insulin $(\mathrm{uU} / \mathrm{ml}) \times$ glucose $(\mathrm{mmol} / \mathrm{l}) / 22.5$ and $\mathrm{HOMA}_{\mathrm{IS}}=(20 \times$ insulin $(\mathrm{uU} / \mathrm{ml})) /($ fasting blood glucose $(\mathrm{mmol} / \mathrm{l})-3.5)$. The percentage of fat $(\mathrm{FAT} \%)$ was calculated with the following equations: $\mathrm{FAT} \%=1.2$ $\times \mathrm{BMI}+0.23 \times$ age -16.2 (male) and $1.2 \times \mathrm{BMI}+$ $0.23 \times$ age -5.4 (female) (13).

Table 1 Clinical characteristics of study subjects. Data are mean \pm s.D. versus NGT group.

\begin{tabular}{|c|c|c|c|}
\hline Group & T2DM & IGR & NGT \\
\hline$n$ (male/female) & $54(29 / 25)$ & $53(25 / 28)$ & $53(26 / 27)$ \\
\hline Age & $54 \pm 11$ & $54 \pm 10$ & $51 \pm 7$ \\
\hline $\mathrm{BMI}\left(\mathrm{kg} / \mathrm{m}^{2}\right)$ & $24.98+3.75$ & $24.70+2.78$ & $24.53+3.66$ \\
\hline FAT (\%) & $31.06 \pm 7.48$ & $30.55 \pm 6.74$ & $30.87 \pm 8.09$ \\
\hline WHR & $0.91 \pm 0.05^{\star}$ & $0.90 \pm 0.08$ & $0.87 \pm 0.08$ \\
\hline $\mathrm{FBG}(\mathrm{mmol} / \mathrm{l})$ & $10.92 \pm 3.80^{\dagger, \S}$ & $6.03 \pm 0.45^{\dagger}$ & $5.41 \pm 0.43$ \\
\hline 2hPBG (mmol/l) & $21.23 \pm 9.74^{\dagger, \S}$ & $8.86 \pm 1.25^{\dagger}$ & $5.93 \pm 0.81$ \\
\hline $\mathrm{f}-\mathrm{INS}(\mathrm{mU} / \mathrm{l})$ & $8.26 \pm 4.25$ & $8.82 \pm 3.05$ & $7.74 \pm 2.85$ \\
\hline 2hINS (mU/l) & $45.89 \pm 38.52^{\dagger, t}$ & $60.48 \pm 36.97^{\dagger}$ & $26.37 \pm 16.72$ \\
\hline $\mathrm{HOMA}_{\mathrm{IR}}$ & $3.79 \pm 1.77^{\dagger, \S}$ & $2.37 \pm 1.06^{\dagger}$ & $1.85+0.66$ \\
\hline $\mathrm{HOMA}_{\mathrm{IS}}$ & $31.04 \pm 28.09^{\dagger, \S}$ & $72.27 \pm 35.24^{\dagger}$ & $88.79 \pm 49.45$ \\
\hline HbAlc (\%) & $8.8+2.4^{\dagger, \S}$ & $5.9+0.4^{\dagger}$ & $5.5+0.4$ \\
\hline $\mathrm{TG}(\mathrm{mmol} / \mathrm{l})$ & $2.08 \pm 1.56^{\dagger}$ & $1.63 \pm 1.02$ & $1.34 \pm 0.68$ \\
\hline TC $(\mathrm{mmol} / \mathrm{l})$ & $5.25 \pm 1.4$ & $4.98 \pm 1.33$ & $4.83 \pm 0.83$ \\
\hline $\mathrm{HDL}-\mathrm{C}(\mathrm{mmol} / \mathrm{l})$ & $1.29 \pm 0.69$ & $1.22 \pm 0.37$ & $1.27 \pm 0.29$ \\
\hline LDL-C (mmol/l) & $3.01+1.09$ & $2.73+0.81$ & $2.69 \pm 0.68$ \\
\hline FFA (mmol/l) & $0.68 \pm 0.31$ & $0.66 \pm 0.47$ & $0.73 \pm 0.51$ \\
\hline
\end{tabular}

${ }^{*} P<0.05,{ }^{\dagger} P<0.01$; versus IGR group, ${ }^{\ddagger} P<0.05,{ }^{\S} P<0.01$. T2DM, type 2 diabetes mellitus; IGR, impaired glucose regulation; NGT, normal glucose tolerance; BMI, body mass index; WHR, waist-to-hip ratio; FAT\%, the percentage of fat; FBG, fasting blood glucose; 2hPBG, $2 \mathrm{~h}$ blood glucose after a glucose load; f-INS, fasting plasma insulin; 2hINS, $2 \mathrm{~h}$ plasma insulin after a glucose load; HOMA ${ }_{I R}$, HOMA of insulin resistance index; HOMA ${ }_{I S}$, HOMA of $\beta$ cell secretion index; TG, triglyceride; TC, total cholesterol; HDL-C, high-density lipoprotein cholesterol; LDL-C, low-density lipoprotein cholesterol; FFAs, free fatty acids. 


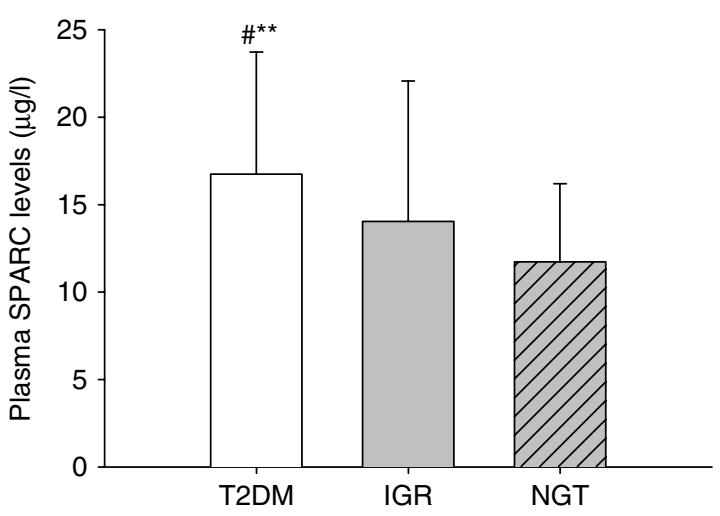

Figure 1 Plasma SPARC levels in three groups. Data are expressed as mean $(\mu \mathrm{g} / \mathrm{l}) \pm$ S.E.M. ${ }^{* \star} P<0.01$ T2DM group versus NGT group and ${ }^{\#} P<0.05$ versus IGR group.

\section{Statistical analysis}

Statistical analyses were performed by SPSS 13.0 Software (SPSS, Inc., Chicago, IL, USA). Data are shown as the mean \pm s.D., with a $P<0.05$ (two-tailed) considered statistically significant. Baseline parameters of the T2DM, IGR, and normal group were compared with each other by one-way ANOVA. The Student's $t$-test was used to compare differences in biochemical characteristics and SPARC between the NW and OW/OB groups. Simple and multiple linear regression analyses were used to examine the association between fasting SPARC levels and the values of other biomarkers. The associations of SPARC with IGR and diabetes were examined by multivariate logistic regression analysis that contains: i) SPARC, age, and gender; ii) SPARC, age, gender, BMI, WHR, and FAT\%; and iii) SPARC, age, gender, BMI, WHR, FAT\%, and lipid profile. The distribution of SPARC in pooled data was further divided into tertiles and analyzed by general linear and logistic regression models to estimate the significant trends across increasing tertile and to estimate the odds ratio (OR) of diabetes in each tertile using the lowest tertile as the reference category. Multivariate adjusted OR is presented with 95\% confidence interval (CI).

\section{Results}

The clinical characteristics of our subjects are shown in Table 1. The subjects with T2DM had higher WHR, TG, fasting blood glucose (FBG), $2 \mathrm{~h}$ blood glucose after a glucose load (2hPBG), 2 h plasma insulin after a glucose

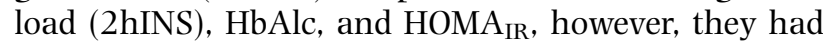
lower HOMA $\mathrm{IS}_{\mathrm{S}}$ than IGR and control subjects $(P<0.01)$. IGR subjects had higher FBG, 2hPBG, 2hINS, HbAlc, and $\mathrm{HOMA}_{\mathrm{IR}}$, and lower HOMA $\mathrm{IS}$ than control subjects. OW/OB subjects had higher FAT\%, BMI, TG, HOMA $\mathrm{A}_{\mathrm{IR}}$, and WHR than NW subjects $(P<0.05)$.
There was no significant difference between male and female subjects in plasma SPARC levels $(13.91 \pm 8.02 \mathrm{vs}$ $14.45 \pm 5.73 \mu \mathrm{g} / \mathrm{l})$. T2DM subjects had higher SPARC levels compared with IGR and control subjects (16.74 \pm 6.99 vs $14.04 \pm 8.03 \mu \mathrm{g} / \mathrm{l}, P<0.05$ and $16.74 \pm 6.99$ vs $11.72 \pm 4.47 \mu \mathrm{g} / \mathrm{l}, P<0.01$; Fig. 1$)$. However, no significant difference in plasma SPARC levels was found between IGR and controls. Subjects with OW/OB had higher plasma SPARC levels than subjects with NW $(15.54 \pm 8.76$ vs $13.01 \pm 4.60 \mu \mathrm{g} / \mathrm{l}, P<0.05)$. Plasma SPARC levels were positively correlated with BMI, FAT $\%$, TG, fasting plasma insulin (f-INS), 2hINS, and $\mathrm{HOMA}_{\mathrm{IR}}$ in simple regression analysis (Table 2). After adjustment for BMI, plasma SPARC levels remained positively correlated with TG $(r=0.17, P<0.05)$, f-INS $(r=0.21, P<0.05)$, and HOMA $\mathrm{IR}(r=0.27, P<0.01)$. Multiple regression analysis showed that $\mathrm{TG}$ and $\mathrm{HOMA}_{\mathrm{IR}}$ were independent factors influencing plasma SPARC levels $\left(Y=1.002 X_{\mathrm{TG}}+1.233 X_{\mathrm{HOMA}_{\mathrm{IR}}}+9.07\right)$.

Multivariate logistic regression analysis showed that plasma SPARC levels were significantly associated with IGR and T2DM even after controlling for gender, WHR, BMI, FAT\%, and lipid profile (Table 3). Increasing levels of SPARC showed a significant linear trend and were independently associated with T2DM, especially when concentrations were analyzed both by tertile and by a continuous variable (Tables 3 and 4). Multiple logistic regression analysis in fully adjusted OR in the second and third tertile were 35.873 (95\% CI, 7.380-174.368) and 45.489 (95\% CI, 9.246-223.815) respectively.

Table 2 Linear and multiple regression analysis of variables associated with plasma secreted protein acidic and rich in cysteine levels in study subjects.

\begin{tabular}{|c|c|c|c|c|}
\hline \multirow[b]{2}{*}{ Variables } & \multicolumn{2}{|c|}{ Simple } & \multicolumn{2}{|c|}{ Multiple } \\
\hline & Estimate & $P$ value & Estimate & $P$ value \\
\hline BMI & 0.190 & 0.016 & - & - \\
\hline FAT\% & 0.204 & 0.010 & - & - \\
\hline WHR & 0.020 & 0.804 & - & - \\
\hline TG & 0.216 & 0.006 & 1.002 & 0.029 \\
\hline $\mathrm{TC}$ & 0.050 & 0.532 & - & - \\
\hline HDL-C & -0.032 & 0.690 & - & - \\
\hline LDL-C & 0.033 & 0.681 & - & - \\
\hline FFA & 0.066 & 0.410 & - & - \\
\hline $\mathrm{HbAlc}$ & 0.132 & 0.095 & - & - \\
\hline FBG & 0.120 & 0.131 & - & - \\
\hline 2hPBG & 0.139 & 0.080 & - & - \\
\hline f-INS & 0.213 & 0.007 & - & - \\
\hline 2hINS & 0.160 & 0.046 & - & - \\
\hline $\mathrm{HOMA}_{\mathrm{IR}}$ & 0.270 & 0.001 & 1.233 & 0.001 \\
\hline $\mathrm{HOMA}_{\mathrm{IS}}$ & -0.046 & 0.565 & - & - \\
\hline
\end{tabular}

In multiple linear stepwise regression analysis, values included for analysis were gender, body mass index (BMI), waist-to-hip ratio (WHR), the percentage of fat (FAT\%), fasting blood glucose (FBG), $2 \mathrm{~h}$ blood glucose after a glucose load (2hPBG), fasting plasma insulin (f-INS), $2 \mathrm{~h}$ plasma insulin after a glucose load (2hINS), HOMA of insulin resistance index $\left(\mathrm{HOMA}_{\mathrm{IR}}\right), \mathrm{HOMA}$ of $\beta$ cell secretion index $\left(\mathrm{HOMA}_{\mathrm{IS}}\right)$, triglyceride $(\mathrm{TG})$, total cholesterol (TC), high-density lipoprotein cholesterol (HDL-C), low-density lipoprotein cholesterol (LDL-C), and free fatty acids (FFAs). 
Table 3 Association of plasma secreted protein acidic and rich in cysteine with IGR and type 2 diabetes in fully adjusted models.

\begin{tabular}{|c|c|c|c|c|c|c|}
\hline \multirow[b]{2}{*}{ Model adjusted for } & \multicolumn{3}{|c|}{ IGR } & \multicolumn{3}{|c|}{ T2DM } \\
\hline & OR & $95 \% \mathrm{Cl}$ & $P$ value & OR & $95 \% \mathrm{Cl}$ & $P$ value \\
\hline Age and gender & 1.789 & $0.542-5.911$ & 0.34 & 28.576 & $6.24-130.863$ & 0.000 \\
\hline Age, gender, BMI, WHR, and FAT\% & 2.447 & $0.661-9.068$ & 0.18 & 38.939 & $7.735-196.022$ & 0.000 \\
\hline $\begin{array}{l}\text { Age, gender, BMI, WHR, FAT\%, TG, } \\
\text { TC, HDL-C, LDL-C, and FFA }\end{array}$ & 2.238 & $0.567-8.842$ & 0.25 & 49.456 & $8.745-279.686$ & 0.000 \\
\hline
\end{tabular}

T2DM, type 2 diabetes mellitus; IGR, impaired glucose regulate; NGT, normal glucose regulate; BMI, body mass index; WHR, waist-to-hip ratio; FAT\%, the percentage of fat; TG, triglyceride; TC, total cholesterol; HDL-C, high-density lipoprotein cholesterol; LDL-C, low-density lipoprotein cholesterol; FFAs, free fatty acids; OR, odds ratio.

\section{Discussion}

SPARC, which has been studied in various pathological conditions is a glycoprotein that modulates tissue physiology by altering the cell-ECM (extracellular matrix) interaction, cell proliferation, and cell migration. Recent evidence suggests that SPARC could be a key player in the pathology of obesity and T2DM. The biological mechanisms involving SPARC in the pathogenesis of IR and T2DM remain poorly understood, although potential mechanisms have been postulated, including the interaction of SPARC and macrophage migration inhibitory factor (MMIF) involved in inflammatory processes (2).

In this study, we observed that the fasting plasma SPARC levels were significantly higher in subjects with T2DM compared with IGR and control subjects. We also found that there was a trend of increasing SPARC levels in IGR subjects compared with controls. To the best of our knowledge, this is the first study to describe plasma levels of SPARC in patients with different glucose regulatory conditions. Although SPARC levels have been demonstrated to be downregulated by glucose in vitro $(2,13,14)$, other modulators, including insulin and leptin, which have been reported as positive modulators, may also result in the elevation of SPARC levels in diabetic subjects. Adipose tissue is known to be an active and dynamic organ expressing and secreting several cytokines and chemokines. However, it is still not clear whether or not plasma SPARC level can be influenced by other adipokines aside from leptin. Our data also demonstrated that $\mathrm{OW}$ and $\mathrm{OB}$ subjects had higher SPARC levels than subjects with NW, in accordance with previous reports (2). Furthermore, SPARC levels were found to correlate positively with BMI, FAT\%, TG, f-INS, 2hINS, and HOMA ${ }_{\text {IR }}$ in simple regression analysis, and multiple regression analysis showed that TG and $\mathrm{HOMA}_{\mathrm{IR}}$ were independently correlated with plasma SPARC levels. As shown in the literature, SPARC has been shown to inhibit the differentiation of preadipocytes to mature adipocytes, and to increase adipose tissue fibrosis $(15,16)$. Thus, SPARC could inhibit the expansion of subcutaneous abdominal adipose tissue (6), reduce the ability of adipose tissue to store TGs, and increase the TGs in the circulation and lipid infiltration of other organs resulting in IR. Multivariate logistic regression analysis showed that plasma SPARC levels were significantly associated with IGR and T2MD even after adjusting for age, gender, WHR, BMI, FAT\%, and lipid profile, further supporting a role for SPARC in the pathogenesis of IR and T2DM. Some limitations of this study should be considered. The FAT\% formula used in our study is validated in males and females of all ages. However, the formula slightly overestimated the body fat percentage in OB subjects with a BMI over 30. Our analyses are based on single measurements of blood SPARC, which may not reflect changes over time. Serial changes in plasma SPARC need to be measured at different stages of T2DM besides new-onset T2DM to further clarify the role of SPARC in the pathogenesis of T2DM. Moreover, it would be interesting to measure related cytokines and peptides such as MMIF, interleukin 1 (17) and tumour necrosis factor (TNF) (18) involved in inflammatory processes and further explore the potential mechanisms between SPARC and T2DM.

Table 4 Univariate and multivariate analysis of the impact of plasma secreted protein acidic and rich in cysteine (SPARC) on type 2 diabetes mellitus. Values shown are cut offs of plasma SPARC levels of all subjects, and OR with $95 \%$ confidence interval (Cl).

\begin{tabular}{|c|c|c|c|c|}
\hline \multirow[b]{2}{*}{ Factor } & \multicolumn{4}{|c|}{ Tertiles of SPARC } \\
\hline & Q1 $(95 \% \mathrm{Cl})$ & Q2 (95\% Cl) & Q3 $(95 \% \mathrm{Cl})$ & $P$ for trend \\
\hline \multicolumn{5}{|l|}{ All subjects } \\
\hline No. of IGR/T2DM/reference & $21 / 3 / 31$ & $17 / 23 / 12$ & $15 / 28 / 10$ & $<0.001$ \\
\hline Cut off SPARC (ng/ml) & $<11.371$ & $11.371-15.024$ & $15.024-57.821$ & \\
\hline Univariate & 1.00 & $19.806(5.006-78.359)$ & $28.933(7.223-115.898)$ & $<0.001$ \\
\hline Multivariate $^{\mathrm{a}}$ & 1.00 & $35.873(7.380-174.368)$ & $45.489(9.246-223.815)$ & $<0.001$ \\
\hline
\end{tabular}

${ }^{a}$ Adjusted for age, sex, body mass index, waist-to-hip ratio, the percentage of fat, total cholesterol, triglyceride, low-density lipoprotein cholesterol, high-density lipoprotein cholesterol, and free fatty acids. 
In conclusion, our results showing increased SPARC in $\mathrm{OB}$ and T2DM subjects suggested that SPARC may play a role in pathogenesis of both obesity and diabetes. However, further studies are needed to clarify the biological mechanisms involving SPARC in the pathogenesis of T2DM.

\section{Declaration of interest}

The authors declare that there is no conflict of interest that could be perceived as prejudicing the impartiality of the research reported.

\section{Funding}

This work was supported by grants from the National Natural Science Foundation of China $(30871199,30771037,30971388$, and 81070640) and Doctoral Fund of Ministry of Education of China (20105503110002).

\section{Acknowledgements}

We thank Dr Linda L Finnegan (G V Montgomery, VA Medical Center, Jackson, MS, USA) for reviewing and editing this manuscript.

\section{References}

1 Termine JD, Kleinman HK, Whitson SW, Conn KM, McGarvey ML \& Martin GR. Osteonectin, a bone-specific protein linking mineral to collagen. Cell $1981 \quad 26$ 99-105. (doi:10.1016/00928674(81)90037-4)

2 Kos K, Wong S, Tan B, Gummesson A, Jernas M, Franck N, Kerrigan D, Nystrom FH, Carlsson LM, Randeva HS, Pinkney JH \& Wilding JP. Regulation of the fibrosis and angiogenesis promoter SPARC/osteonectin in human adipose tissue by weight change, leptin, insulin, and glucose. Diabetes 200958 1780-1788. (doi:10.2337/db09-0211)

3 Sage H, Johnson C \& Bornstein P. Characterization of a novel serum albumin-binding glycoprotein secreted by endothelial cells in culture. Journal of Biological Chemistry 1984259 3993-4007.

4 Reed MJ \& Sage EH. SPARC and the extracellular matrix: implications for cancer and wound repair. Current Topics in Microbiology and Immunology 1996213 81-94.

5 Bradshaw AD, Reed MJ \& Sage EH. SPARC-null mice exhibit accelerated cutaneous wound closure. Journal of Histochemistry and Cytochemistry $2002 \quad 50 \quad 1-10 . \quad$ (doi:10.1177/002215540 205000101)

6 Camino AM, Atorrasagasti C \& Maccio D. Adenovirus-mediated inhibition of SPARC attenuates liver fibrosis in rats. Journal of Gene Medicine 200810 993-1004. (doi:10.1002/jgm.1228)

7 Bradshaw AD, Graves DC, Motamed K \& Sage EH. SPARC-null mice exhibit increased adiposity without significant differences in overall body weight. PNAS $2003 \mathbf{1 0 0}$ 6045-6050. (doi:10. $1073 /$ pnas.1030790100)
8 Tartare-Deckert S, Chavey C, Monthouel MN, Gautier N \& Van Obberghen E. The matricellular protein SPARC/osteonectin as a newly identified factor up-regulated in obesity. Journal of Biological Chemistry $2001 \quad 276 \quad 22231-22237 . \quad$ (doi:10.1074/jbc. M010634200)

9 Gilbert RE, McNally PG, Cox A, Dziadek M, Rumble J, Cooper ME \& Jerums G. SPARC gene expression is reduced in early diabetesrelated kidney growth. Kidney International 199548 1216-1225. (doi:10.1038/ki.1995.405)

10 Taneda S, Pippin JW, Sage EH, Hudkins KL, Takeuchi Y, Couser WG \& Alpers CE. Amelioration of diabetic nephropathy in SPARC-null mice. Journal of the American Society of Nephrology 200314 968-980. (doi:10.1097/01.ASN.0000054498.83125. 90)

11 Watanabe K, Okamoto F, Yokoo T, Iida KT, Suzuki H, Shimano H, Oshika T, Yamada N \& Toyoshima H. SPARC is a major secretory gene expressed and involved in the development of proliferative diabetic retinopathy. Journal of Atherosclerosis and Thrombosis 2009 16 69-76. (doi:10.5551/jat.E711)

12 Munjal ID, McLean NV, Grant MB \& Blake DA. Differences in the synthesis of secreted proteins in human retinal endothelial cells of diabetic and nondiabetic origin. Current Eye Research 199413 303-310. (doi:10.3109/02713689408995792)

13 Deurenberg P, Weststrate JA \& Seidell JC. Body mass index as a measure of body fatness: age- and sex-specific prediction formulas. British Journal of Nutrition 199165 105-114. (doi:10.1079/ BJN19910073)

14 Mansergh FC, Wells T, Elford C, Evans SL, Perry MJ, Evans MJ \& Evans BA. Osteopenia in SPARC (osteonectin)-deficient mice: characterization of phenotypic determinants of femoral strength and changes in gene expression. Physiological Genomics 200732 64-73. (doi:10.1152/physiolgenomics.00151.2007)

15 Nie J \& Sage EH. SPARC inhibits adipogenesis by its enhancement of beta-catenin signaling. Journal of Biological Chemistry 2009284 1279-1290. (doi:10.1074/jbc.M808285200)

16 Kos K \& Wilding JP. SPARC: a key player in the pathologies associated with obesity and diabetes. Nature Reviews. Endocrinology 20106 225-235. (doi:10.1038/nrendo.2010.18)

17 Alvarez MJ, Prada F, Salvatierra E, Bravo AI, Lutzky VP, Carbone C, Pitossi FJ, Chuluyan HE \& Podhajcer OL. Secreted protein acidic and rich in cysteine produced by human melanoma cells modulates polymorphonuclear leukocyte recruitment and antitumor cytotoxic capacity. Cancer Research 200565 5123-5132. (doi:10.1158/0008-5472.CAN-04-1102)

18 Shiba H, Fujita T, Doi N, Nakamura S, Nakanishi K, Takemoto T, Hino T, Noshiro M, Kawamoto T, Kurihara H \& Kato Y. Differential effects of various growth factors and cytokines on the syntheses of DNA, type I collagen, laminin, fibronectin, osteonectin/secreted protein, acidic and rich in cysteine (SPARC), and alkaline phosphatase by human pulp cells in culture. Journal of Cellular Physiology $1998 \quad \mathbf{1 7 4} 194-205 . \quad$ (doi:10.1002/(SICI)10974652(199802)174:2<194::AID-JCP7> 3.0.CO;2-J)

Received 10 February 2011

Revised version received 15 July 2011

Accepted 21 July 2011 